\title{
Bloch State Contributions to Dopant Atom Contrast in HAADF STEM
}

\author{
B.G. Mendis*
}

* Department of Physics, Durham University, South Road, Durham, DH1 3LE, UK

Modern aberration corrected STEM microscopes enable single atom detection through techniques such as high angle annular dark field (HAADF) imaging [1-3]. Furthermore with large objective aperture sizes optical sectioning experiments can be carried out to determine the distribution of dopant atoms along the specimen thickness direction. For such experiments to be truly quantitative it is important to understand the scattering mechanisms of the STEM probe intensity by the dopant atom. In this paper the scattering mechanisms are examined using Bloch waves [4], which provide greater physical insight compared to the multislice approach.

Bloch wave solutions for a defect crystal containing small elastic strain fields are given by the Howie-Whelan equations and column approximation [4]. However, for a dopant atom there is also a change in the local electrostatic potential, which cannot be modeled using the Howie-Whelan equations. Time-dependent perturbation theory has been applied to determine the Bloch wave solution for a defect crystal containing a change in the local electrostatic potential, i.e. an 'excess' potential ' $v$ ' [5]:

$$
\frac{d \varepsilon^{q}\left(\mathbf{k}_{t}, z\right)}{d z}=\frac{2 \pi i m e}{h^{2} k_{z}} \sum_{p} \varepsilon^{p}\left(\mathbf{k}_{t}, z\right) \exp \left[2 \pi i\left(\gamma^{p}-\gamma^{q}\right) z\right] \int b^{p}\left(\mathbf{k}_{t}, \mathbf{R}\right) v(\mathbf{R}, z) b^{q}\left(\mathbf{k}_{t}, \mathbf{R}\right) * d \mathbf{R}
$$

where $\varepsilon^{q}$ is the excitation of the $q^{\text {th }}$ Bloch state $b^{q}, \gamma^{q}$ is the change in the longitudinal wavevector of the Bloch state due to channeling and $(\mathbf{R}, z)$ are the spatial coordinates perpendicular and parallel to the electron-optic axis respectively. Furthermore $\left(\mathbf{k}_{t}, k_{z}\right)$ are the transverse and longitudinal wavevector components of the incident electron and the * symbol represents the complex conjugate. The summation is carried out over all Bloch states $p$. For a substitutional atom $v$ is equal to the difference in potential between the dopant and host atom while for an interstitial atom it is equal to the dopant atom potential. Thermal diffuse scattering (TDS) is modeled phenomenologically by introducing an imaginary term in $v$. In a HAADF STEM calculation the above equation is applied to each partial plane wave within the STEM probe (see [6] for more details).

Bloch wave calculations are carried out for a $100 \AA$ thick, [111]-oriented Fe specimen containing a W substitutional dopant atom at depths of 18, 46 and $66 \AA$ respectively. The STEM probe semiconvergence angle is $20 \mathrm{mrad}$ and the microscope voltage is $300 \mathrm{kV}$. All electron optic aberrations are set to zero. Under these conditions depths of 18 and $66 \AA \AA$ correspond to maxima in the intensity of a STEM probe positioned over an atom column, while a depth of $46 \AA$ corresponds to an intensity minimum. Fig. 1(a) shows the calculated change in the STEM probe intensity as a function of depth along the atom column containing a W substitutional atom. The STEM probe is incident on the atom column containing the $\mathrm{W}$ dopant atom and the change in intensity is with respect to a perfect crystal. There is an intensity valley ' $A$ ' at the dopant atom followed by a peak labeled ' $B$ '. These features can be explained by examining the $1 \mathrm{~s}$ and non-1s Bloch state contributions to the STEM probe intensity [6]. The change in STEM probe intensity due to the 1s and non-1s Bloch states are plotted in Figs. 1(b) and 1(c) respectively. The 1s Bloch state channels along the atom columns and undergoes enhanced TDS by the heavier W atom. This leads to a decrease in 1s intensity at the 
dopant atom (Fig. 1(b)) and hence the intensity valley 'A' in Fig. 1(a). The decrease in 1s intensity due to TDS is dependent on the depth of the dopant atom, such that it is smallest for the W atom at $46 \AA$, where the STEM probe intensity is at a minimum. This is consistent with high angle scattering varying monotonically with the local electron beam intensity, as expected for HAADF imaging. The non-1s Bloch states, which are largely responsible for the probe intensity surrounding the dopant atom, are Coulomb attracted towards the higher atomic number $\mathrm{W}$ atom. Hence there is an increase in the non-1s intensity close to the dopant atom (Fig. 1(c)), giving rise to the peak 'B' in Fig. 1(a). Peak 'B' represents an 'atom focusing effect' that is similar to channeling of the STEM probe along an atom column. Frozen phonon, multislice simulations (Fig. 1(d)) have also been carried out to determine the accuracy of the Bloch wave calculations. The gross features in Fig. 1(a) have been qualitatively reproduced apart from the intensity valley ' $\mathrm{A}$ '. This could be due to 'noise' from the limited number of frozen phonon configurations used to generate Fig. 1(d).

References

[1] K. van Benthem et al., Appl. Phys. Lett. 87 (2005), 034104-1.

[2] P.M. Voyles et al., Nature 416 (2002), 826.

[3] J.E. Allen et al., Nature Nanotech. 3 (2008), 168.

[4] P.B. Hirsch et al., Electron Microscopy of Thin Crystals, Washington: Butterworths, 1965.

[5] B.G. Mendis, Acta Cryst. A 64 (2008), 613.

[6] B.G.Mendis, Acta Cryst. A, in press.

[7] BGM would like to thank the Dept. of Physics, Durham University for financial support.
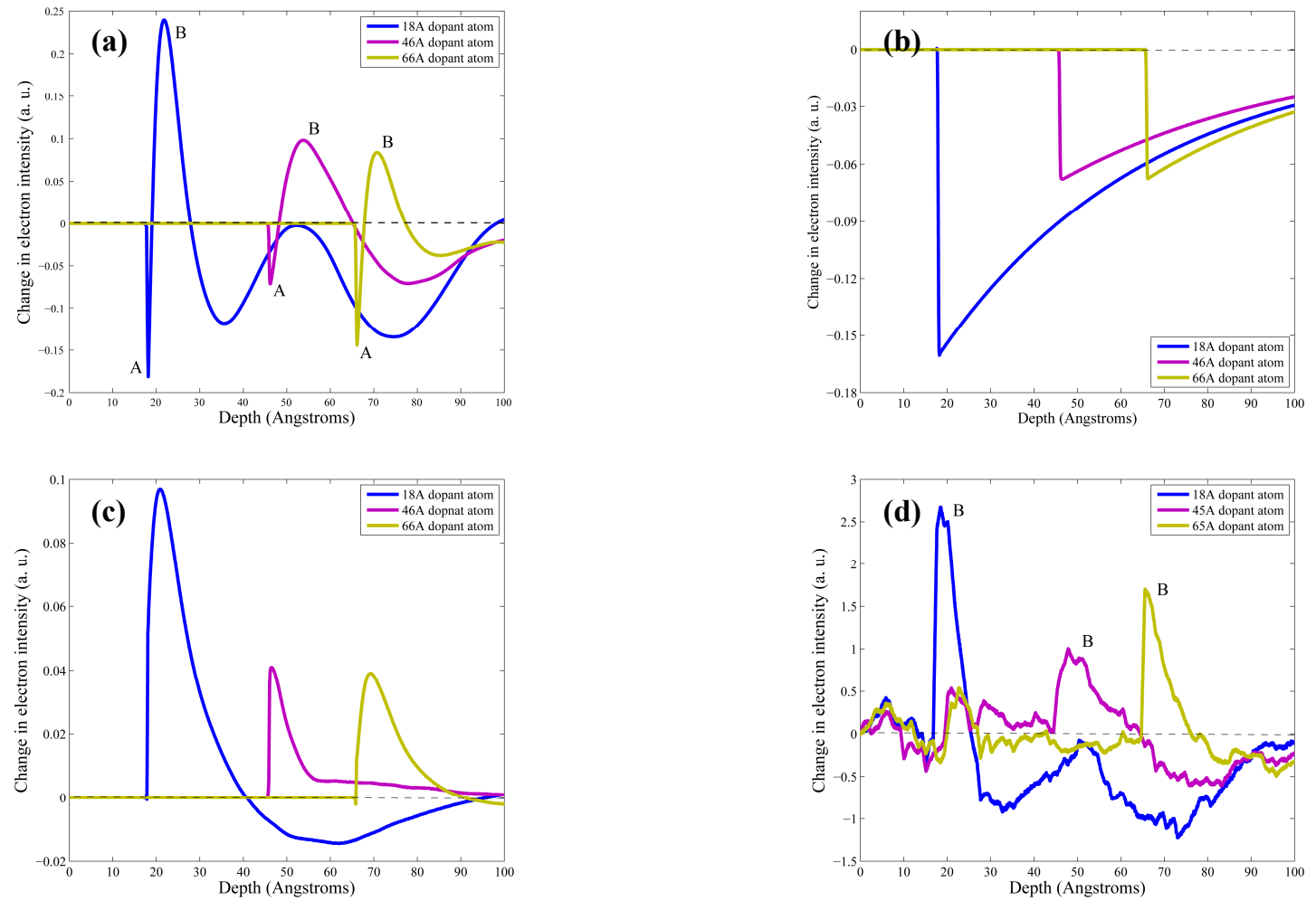

FIG 1: Change in STEM probe intensity with depth: (a) full Bloch wave calculation, (b) 1s contribution, (c) non-1s contribution and (d) multislice simulation. 